\title{
LAS TERAPIAS NO CONVENCIONALES EN LA CAMPAÑA DE LAS ELECCIONES GENERALES DE 2015
}

\section{Complementary and alternative medicine therapies in the General Elections campaign of 2015}

\author{
Lorena Cano Orón ${ }^{1}$, Carolina Moreno Castro
}

Universidad de Valencia

\section{Resumen}

La presente investigación tiene como objetivo valorar y estudiar el tratamiento de las terapias no convencionales, complementarias, alternativas o integrativas a través de los programas electorales de las Elecciones Generales de 2015 de cinco partidos políticos: Ciudadanos, Podemos, PP, PSOE y Unidad Popular. Asimismo, se analizan los tuits publicados por sus cabezas de lista, durante la campaña electoral. Para abordar la investigación, hemos recurrido a dos métodos de recogida de datos y su posterior evaluación. Para estudiar los programas electorales, hemos utilizado una ficha de vaciado de análisis de contenido. Para analizar los tuits, hemos empleado el software T-LAB 9.1. de análisis de corpus lingüístico. Los principales resultados de la investigación muestran que se hacen muy pocas referencias a este tipo de terapias. En Unidad Popular y Ciudadanos proponen que no formen parte de la cartera de servicios del SNS, mientras que el PSOE propone regularlas para aquellas personas que deseen utilizarlas.

Palabras clave: Programa electoral, sanidad, terapias no convencionales, elecciones generales 2015, Twitter.

\begin{abstract}
This research aims to evaluate and study the treatment of non-conventional therapies, complementary, alternative or integrative through the electoral programs of the general elections of 2015 of five political parties: Ciudadanos, PP, PSOE and Unidad Popular. Also, the tweets published by their chief candidates during the election campaign are analyzed. To address the research, we have used two methods of data collection and evaluation. To study the electoral programs, we used a file dump content analysis. To analyze tweets, we have used the T-LAB 9.1 software corpus linguistic analysis. The main results of the research show that very few references to such therapies are made. Unidad Popular and Ciudadanos propose that are not part of the service portfolio of the SNS, while the PSOE proposes to regulate for those wishing to use them.
\end{abstract}

Keywords: Electoral program, health, CAM therapies, general elections 2015, Twitter

\footnotetext{
${ }^{1}$ Autor de contacto: Lorena Cano Orón Lorena.Cano@uv.es
} 


\section{Cómo citar el artículo}

Cano Orón, L., Moreno Castro, C. (2017). Las terapias no convencionales en la campaña de las elecciones generales de 2015. Revista de Comunicación y Salud, vol. $7, \quad$ pp. 29-47. Recuperado de http://revistadecomunicacionysalud.org/index.php/rcys/article/view/111

El presente trabajo ha sido desarrollado en el marco del proyecto de investigación "Estudio y clasificación de las terapias naturales, complementarias y alternativas a través de los medios de comunicación y de las redes sociales. Ideas y valores de transferencia al imaginario social" (CSO2014-57778-R), financiado por el Ministerio de Economía y Competitividad y por fondos FEDER de la Unión Europea.

\section{INTRODUCCIÓN}

Se estima que la sanidad pública ha perdido 10.000 millones de euros durante el periodo de la crisis económica ${ }^{1}$. Muchos pacientes crónicos, así como población geriátrica, especialmente, han visto afectada sus prestaciones, por los recortes llevados a cabo en las políticas públicas de Sanidad (Idoyaga, 2000). Esta es una de las razones por las que algunos pacientes deciden incorporar las medicinas alternativas a sus procesos de curación. Precisamente los pacientes con mayor nivel educativo y, por tanto, con mayor acceso a la información son los que recurren con mayor frecuencia a estas terapias, en las que se hace especial hincapié en los estilos de vida saludables y en la promoción de la salud. Según Ballvé (2003), "el profesional de la salud se convierte en un asesor, con una nueva función consistente en orientar a los pacientes para que escojan e interpreten correctamente la información sobre la salud que se encuentra disponible en todos los medios: radio y televisión, prensa, libros o Internet". Este autor, reflexiona sobre el empoderamiento de los pacientes en la medicina alternativa, ya que considera que los pacientes estarán preparados para participar en la toma de decisiones sobre su salud de forma adulta y responsable; siempre apoyados y orientados por su médico. En esta línea, otro de los motivos de esta reflexión está relacionado con las enfermedades crónicas, las enfermedades de mal pronóstico y los pacientes somatizadores, dado que los éxitos de la medicina convencional, espectacularizados, a veces de forma excesiva, por los medios de comunicación y por el marketing de la propia industria farmacéutica, pueden dificultar la aceptación de una realidad no tan maravillosa por parte de muchos pacientes.

En España sigue vigente el debate sobre la efectividad y regulación de las terapias no convencionales. Actualmente, se trata de un sector sanitario que se encuentra en un limbo de a-legalidad ${ }^{2}$. Según datos oficiales de 2011, están registradas 139 técnicas como terapias naturales y su aplicación, tanto en el ámbito español como en el europeo, está en aumento a pesar de tener una escasa incorporación a la sanidad pública en el caso internacional y nula en el caso español (Ministerio de Sanidad, Política Social e Igualdad, 2011). Como bien señala el informe oficial, existe un riesgo para el usuario que acude a las terapias no convencionales por temas de salud, pues "algunas terapias naturales presentan riesgo de daño y efectos adversos, a veces severos, debidos a las características del producto utilizado o de la propia técnica o procedimiento (invasividad), a la indicación o a la utilización inapropiada, a la inadecuada experiencia de los terapeutas, o al retraso en el acceso al tratamiento convencional eficaz demostrado 
científicamente para la situación clínica del paciente." (Ministerio de Sanidad, 2011: 73)

Como detectó el Ministerio de Sanidad en 2011 (p.74), la tendencia en España de la utilización de estas técnicas, su divulgación y su publicidad está en aumento. Es fuertemente sorprendente que mientras que la ciencia no aprueba estas terapias, una gran mayoría de universidades oferta enseñanzas propias, como cursos de postgrado especializados, desde hace más de dos décadas, normalmente clasificadas dentro del ámbito de ciencias de la salud.

Han sido los gobiernos del PSOE y del PP, los agentes activos en la historia de la regulación de las terapias no convencionales. En 1994, con Felipe González como presidente del gobierno, surge la primera regulación del sector ${ }^{3}$, que es la que permitía continuar en el mercado a los productos homeopáticos, que ya estaban siendo comercializados, pero tenían que dirigirse al Ministerio de Sanidad para solicitar la autorización y realizar el registro. En 2003, con José María Aznar de presidente, se desarrolló un decreto ${ }^{4}$ que contemplaba las terapias no convencionales como unidad asistencial integrada en los centros sanitarios, dependiendo en este caso de la autoridad sanitaria de la comunidad autónoma correspondiente.

En 2007, con José Luis Rodríguez Zapatero como presidente, se creó un grupo de trabajo en el Ministerio de Sanidad para investigar el estado de las terapias naturales en España ${ }^{5}$. En 2013, siendo Mariano Rajoy presidente, se publica un borrador $^{6}$ de una orden ministerial que regularía 19.000 fármacos homeopáticos. A pesar de que se creía que a finales de 2014 la regulación sobre los productos homeopáticos sería aprobada, a fecha de marzo de 2016, sigue paralizada.

En el caso concreto de las terapias no convencionales, es muy significativa la postura que adopten los políticos y los partidos. Existe ya un debate público que cuestiona la utilización y el empleo de estas terapias por la población. De hecho, algunos agentes sociales consideran que deberían ser reguladas normativamente e integradas en la sanidad pública. Puesto que cualquier decisión al respecto afectaría también al concepto de sanidad pública española, creemos altamente interesante estudiar este tema dentro del periodo de campaña electoral.

El uso de las TIC ha supuesto una transformación de la esfera pública, "históricamente monopolizada por unos pocos actores sociales que ostentaban una capacidad casi total para controlar el flujo de información y los procesos de formación de opinión en las sociedades contemporáneas" (Valera, 2014, p. 154). Redes sociales como Twitter y Facebook son generadoras de noticias y son capaces de marcar agenda en la prensa tradicional. De hecho, "los medios sociales son un barómetro de influencia y los medios crecen en difusión gracias a las redes sociales al existir una promoción multiplataforma" (Palomo, 2014, p. 112).

El objetivo principal de esta investigación es analizar el papel que los partidos políticos le han atribuido a la sanidad, concretamente a las terapias no convencionales, en las elecciones generales de 2015 en sus programas electorales y a través del discurso digital generado en Twitter por sus cabezas de lista. La elección de Twitter para analizar el discurso digital de los cabezas de lista es porque abordan temas en los que se quieren distinguir del resto de partidos. Además, características propias de la red como su gran capacidad de difusión y condensación de mensajes "convierten a Twitter en un instrumento idóneo para que los líderes de opinión traten de marcar la agenda del ámbito en el que operan” (López García et al. 
2015, p. 217). Asimismo estos mensajes también contribuyen a construir la reputación online de los partidos, líderes y representantes políticos y sus interacciones con los agentes de toma de decisiones en políticas públicas (Cuesta, Ugarte, Gaspar y Menéndez, 2011; Cuesta y Gaspar, 2013). Sin embargo, siendo conscientes de la limitación del presente medio para poder desarrollar las propuestas de campaña en su amplitud, se analiza también el programa electoral, donde cada partido refleja sus motivaciones y preocupaciones en la sociedad española actual.

Las tres hipótesis de las que partía esta investigación fueron: 1) En el discurso generado en Twitter por los cabezas de lista de los partidos seleccionados, la sanidad tendría un protagonismo suficiente como para ser considerada un punto de su agenda temática, debido a que es uno de los pilares fundamentales de la sociedad. 2) En Twitter, serían los candidatos de los partidos más cercanos a la derecha política los que tendrían una cuota de tratamiento del tema sanitario inferior a la del resto, por ser los precursores y defensores de la ejecución de recortes en sanidad. Mientras que los partidos más escorados hacia la izquierda política serían los que publicarían un mayor número de mensajes sobre el estado de la sanidad en España. 3) La manifestación de los candidatos respecto a las terapias no convencionales tendría una representación mínima o nula en el discurso digital, pero esto se contrarrestaría con un desarrollo amplio en política sanitaria en el programa electoral del partido.

\section{MATERIAL Y MÉTODO}

\subsection{La selección de la muestra}

El corpus de esta investigación está compuesto por los programas electorales de las elecciones generales españolas, celebradas el 20 de diciembre de 2015, de cinco formaciones políticas (Partido Popular, Partido Socialista Obrero Español, Podemos, Ciudadanos y Unidad Popular) y los tuits emitidos durante el periodo de campaña electoral por sus cabezas de lista: Mariano Rajoy, Pedro Sánchez, Pablo Iglesias, Albert Rivera y Alberto Garzón.

Los tuits han sido obtenidos mediante la aplicación Twitonomy ${ }^{7}$. Se recogieron todos los tuits publicados por los candidatos políticos entre las $00.00 \mathrm{~h}$ del día 4 de diciembre, inicio de la campaña electoral, y las 23.59h del día 18 de diciembre, cierre de la campaña. El total de mensajes recopilados asciende a 2.226 tuits [Mariano Rajoy: 632 tuits, Pedro Sánchez: 595 tuits, Pablo Iglesias: 209 tuits, Albert Rivera: 412 tuits, Alberto Garzón: 378 tuits].

Se escogen estos partidos políticos en concreto por ser los que presentan mayor intención de voto, según las encuestas de demoscopia, y también los más votados como representantes para el Congreso ${ }^{8}$. Asimismo, se escogen sus propuestas electorales porque delimitan los objetivos $y$, por ende, la base de las políticas públicas que se llevarían a cabo en el próximo periodo electoral, si obtuvieran el poder suficiente para ello.

Hemos tomado como referencia la voz de los cabezas de lista representantes de los partidos en Twitter ${ }^{9}$. Hemos escogido esta vía porque son más los mensajes que se publican y porque facilita a los partidos políticos el hacerse eco de distintos colectivos que les aludan directamente, así como secundar diversas iniciativas. 


\subsection{Método: Análisis de contenido y análisis de corpus lingüístico}

En el presente trabajo hemos aplicado dos métodos de registro complementarios. En primer lugar, aplicamos un análisis de contenido utilizando el software T-Lab, que permite llevar a cabo un análisis computerizado del corpus lingüístico, aplicando de forma automática el test estadístico chi-cuadrado.

El tipo de análisis específico que se ha ejecutado con T-Lab es, dentro de la variedad de análisis de co-ocurrencias, la comparación entre parejas de palabras clave $^{10}$. Esta función permite comparar los conjuntos de fragmentos ${ }^{11}$ en los que las dos palabras clave seleccionadas están presentes. El resultado se muestra en forma de histograma (ver gráficas 2-8), en el que se presentan a la izquierda aquellos lemas que co-ocurren con las palabras seleccionadas para el análisis. La escala mostrada en la parte superior corresponde con el número de repeticiones en las que cada palabra co-ocurre con los términos. Esta representación gráfica facilita la interpretación de los resultados obtenidos (Yau, 2011; Cairo, 2013).

Esta técnica sería aplicada a los tuits publicados durante la campaña electoral por los cabezas de lista de las cinco formaciones políticas españolas. Concretamente, se ha desarrollado un análisis de co-ocurrencia de las palabras clave "sanidad" y "educación", puesto que son los dos sistemas públicos que más afectados han estado por los recortes presupuestarios y más relación tienen con las terapias no convencionales, ya que son agentes que influyen en la consideración positiva o negativa de estas.

En segundo lugar, se ha llevado a cabo un análisis de contenido de los programas electorales de las cinco formaciones políticas seleccionadas utilizando para ello fichas de vaciado. Esta tenía en cuenta aspectos formales [a) el número total de páginas del programa, b) el orden o relevancia que se le daba a la sección de sanidad, c) el número de páginas dedicadas a las cuestiones sanitarias y d) el porcentaje respecto al total del programa que estas representaban] y aspectos de contenido [e) cuáles de los siguientes campos se trataba en el programa: privatización, servicio público, cuidados paliativos, regulación, financiación, medicina preventiva, profesionales sanitarios, investigación y terapias no convencionales y f) especificando, en el caso de que fuesen abordadas, la nomenclatura con que estos partidos se referían a ellas].

Con este estudio se pretende determinar qué relevancia tienen la sanidad y la medicina preventiva en los programas de salud pública durante este periodo electoral y cómo es abordada esta en los programas electorales por cada uno de los partidos políticos.

\section{RESULTADOS}

\subsection{Análisis de los tuits de los cabezas de lista de los cinco partidos más votados}

Desde una perspectiva general, aplicando el análisis de co-ocurrencia de palabras "Sanidad" y "Alternativa" al conjunto global de mensajes de todos los políticos estudiados, nos encontramos ante una ausencia total de lemas que co-ocurran ambas palabras. Es decir, en ningún momento se ha planteado en discurso de los políticos analizados una sanidad alternativa o una alternativa a cualquier aspecto de la sanidad. Como se puede comprobar en el gráfico 1, las palabras que más destacan por su número elevado de ocurrencias con "sanidad" son "educación", "público", "derecho", "PP" y "gobierno". Mientras que en el caso de "alternativa" 
encontramos un número menor de ocurrencias, siendo la que más aparece "PP" seguida de "único".

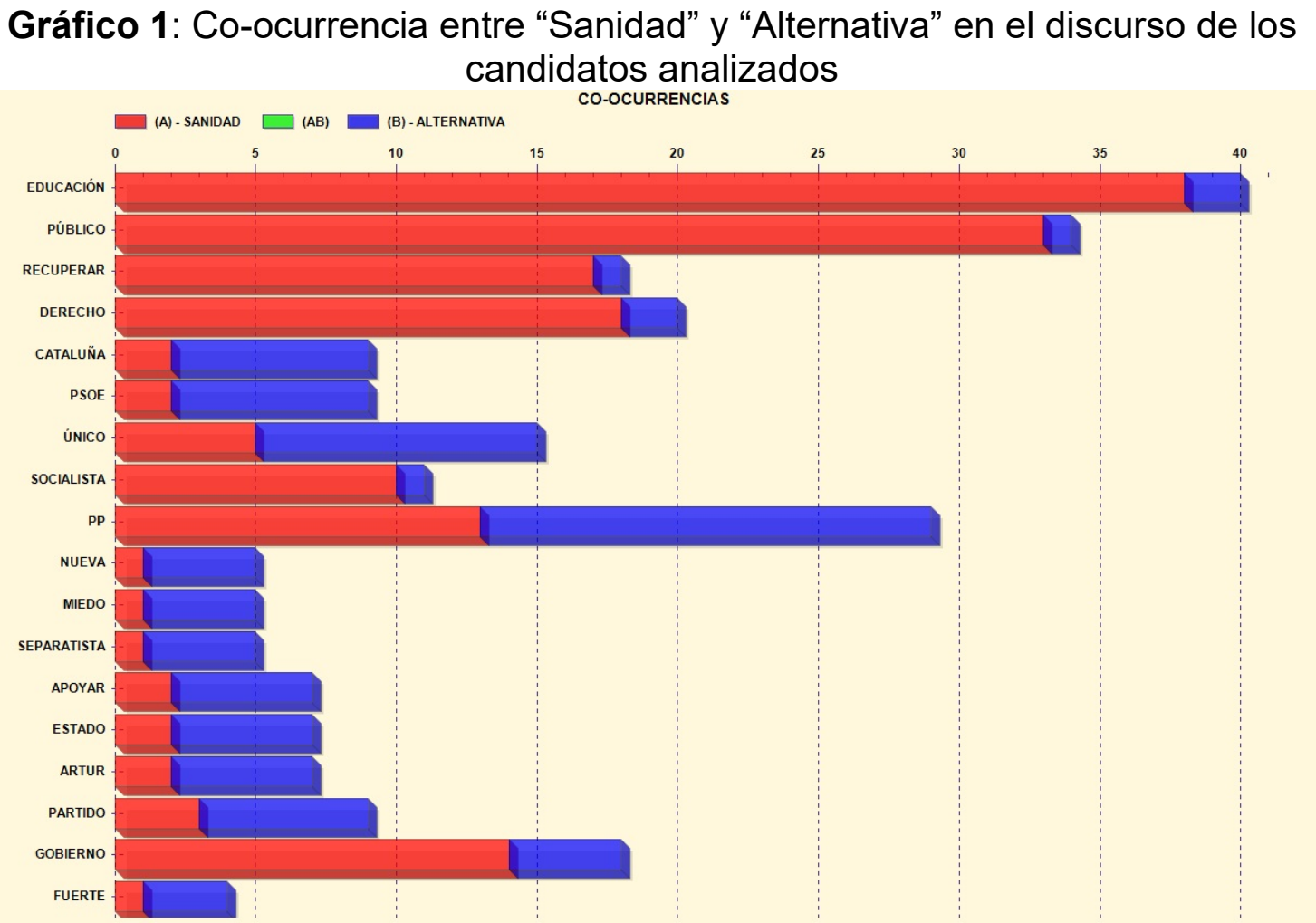

Fuente: Elaboración propia.

Precisamente por la ausencia de elementos co-ocurrentes conjuntamente con los lemas "sanidad" y "alternativa" y por la gran relación entre "sanidad" y "educación", el resto de análisis individualizados de los mensajes de los candidatos se realiza a este segundo conjunto de palabras clave. Como se puede ver en el gráfico 2, las palabras que más aparecen en los discursos de los candidatos relacionadas en los contextos que coinciden con "sanidad" y "educación" son "público", "dependencia" y "derechos". En el caso de los términos co-ocurrentes con "sanidad", destaca "público", como la palabra más usada relacionada con el término, "universal" y "recuperar". Se puede interpretar a partir de estos datos que el discurso de los políticos, cuando hablaban de sanidad, iba siempre relacionada con la idea de servicio público, con una garantía universal, el derecho como ciudadanos a la asistencia sanitaria y la recuperación del sistema sanitario al estado que tenía antes de los recortes aplicados en la última legislatura. 
Gráfico 2: Co-ocurrencia entre "Sanidad" y "Educación" en el discurso de los candidatos analizados

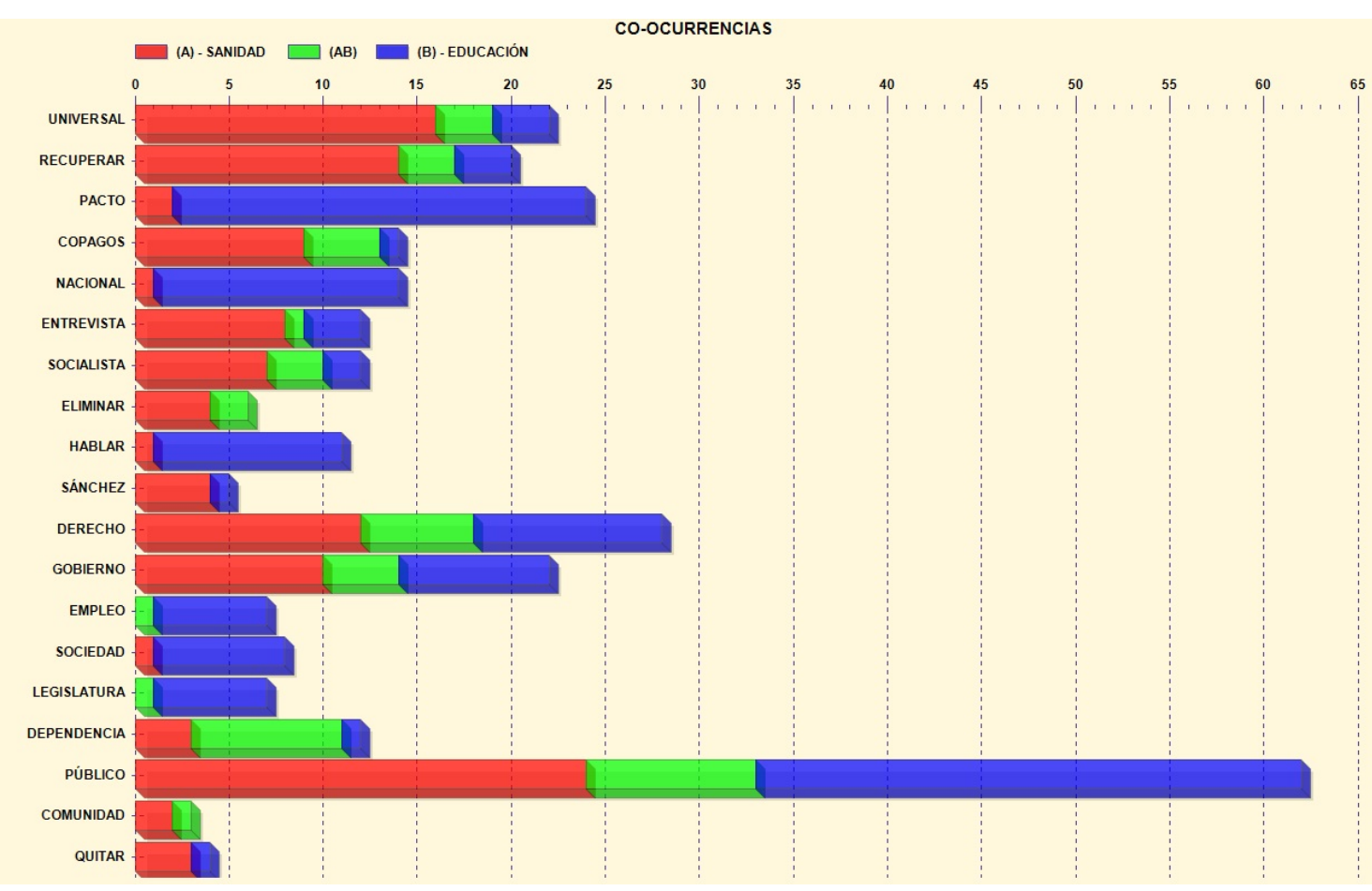

Fuente: Elaboración propia

Es necesario destacar también la ausencia de co-relación de la palabra "sanidad" con "legislatura". Es decir, que no se ha planteado en el discurso de ningún candidato la legislación de la sanidad, y por ende, por lo que a nuestro tema compete, no se ha presentado en el discurso una legislación de las terapias no convencionales.

A continuación nos centramos en el análisis individualizado del discurso de cada candidato para poder realizar comparaciones entre ellos. En el gráfico 3, observamos el histograma resultante al análisis del candidato del Partido Popular, Mariano Rajoy, presidente de la legislatura anterior. Con un total de 10 términos que co-ocurren con las palabras clave "sanidad" y "educación", de forma visual, destaca la escasa importancia que tiene la "sanidad" en su discurso por sí sola. Representada de color rojo, se aprecia cómo sólo es aparecida en cuatro ocasiones, siendo los términos (de mayor a menor frecuencia de repetición) "gobierno" (2 veces), "mejor" (1 vez), "público" (1 vez) y "calidad" (1 vez). En general, los lemas "sanidad" y "educación" han tenido una frecuencia escasa de aparición, siendo la palabra "calidad" la que más co-ocurrencias presenta, sumando un total de 5 entre las tres categorías analizadas. 
Gráfico 3: Co-ocurrencia entre "Sanidad" y "Educación” en el discurso de Mariano Rajoy

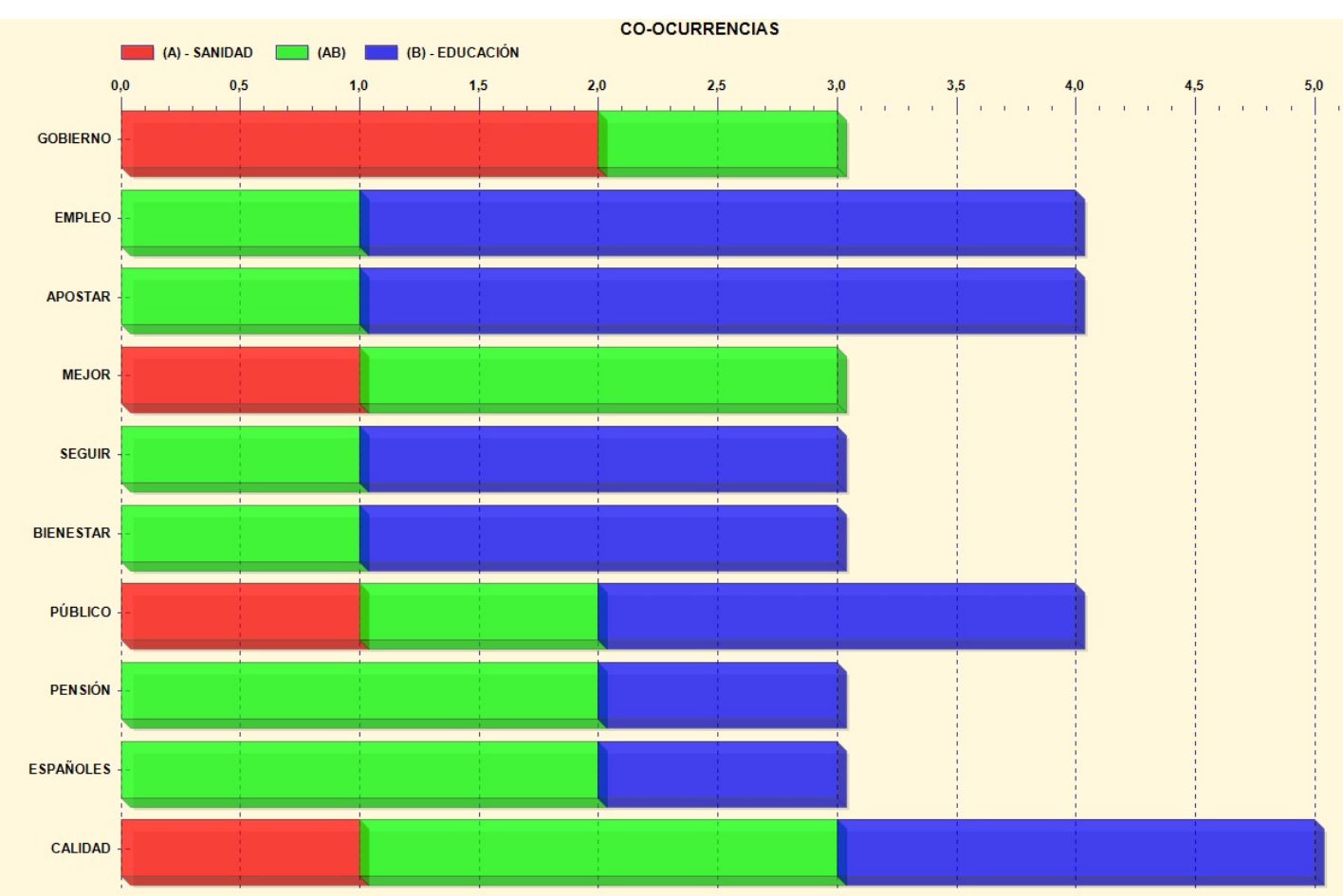

Fuente: Elaboración propia

En cuanto al discurso de Pedro Sánchez, candidato del PSOE, encontramos un total de 18 términos que co-ocurren con "sanidad" y "educación", de los cuales 16 términos tienen relación directa con "sanidad" (gráfico 4). Los lemas con más número de repeticiones relacionadas con "sanidad" son "universal" (14 veces), "recuperar" (13 veces) y "copagos" (9 veces). Es significativo que las palabras coocurrentes "reivindicar" y "someter" tengan mayor número de apariciones con "sanidad" que con "educación" (frecuencia de 3 veces sanidad y 1 educación, respectivamente en ambas); mientras que en el caso de "sociedad", "igualdad", "oportunidad", "mejor" y "PIB" es la vinculación con "educación" la que tiene más peso. Con estos resultados, podemos afirmar que en el discurso llevado a cabo por Pedro Sánchez para hablar sobre sanidad, la reivindica como una universal, suponemos que, sin copagos, por la relación con la palabra "recuperar", que hace referencia al modelo anterior. 
Gráfico 4: Co-ocurrencia entre "Sanidad" y "Educación" en el discurso de Pedro Sánchez

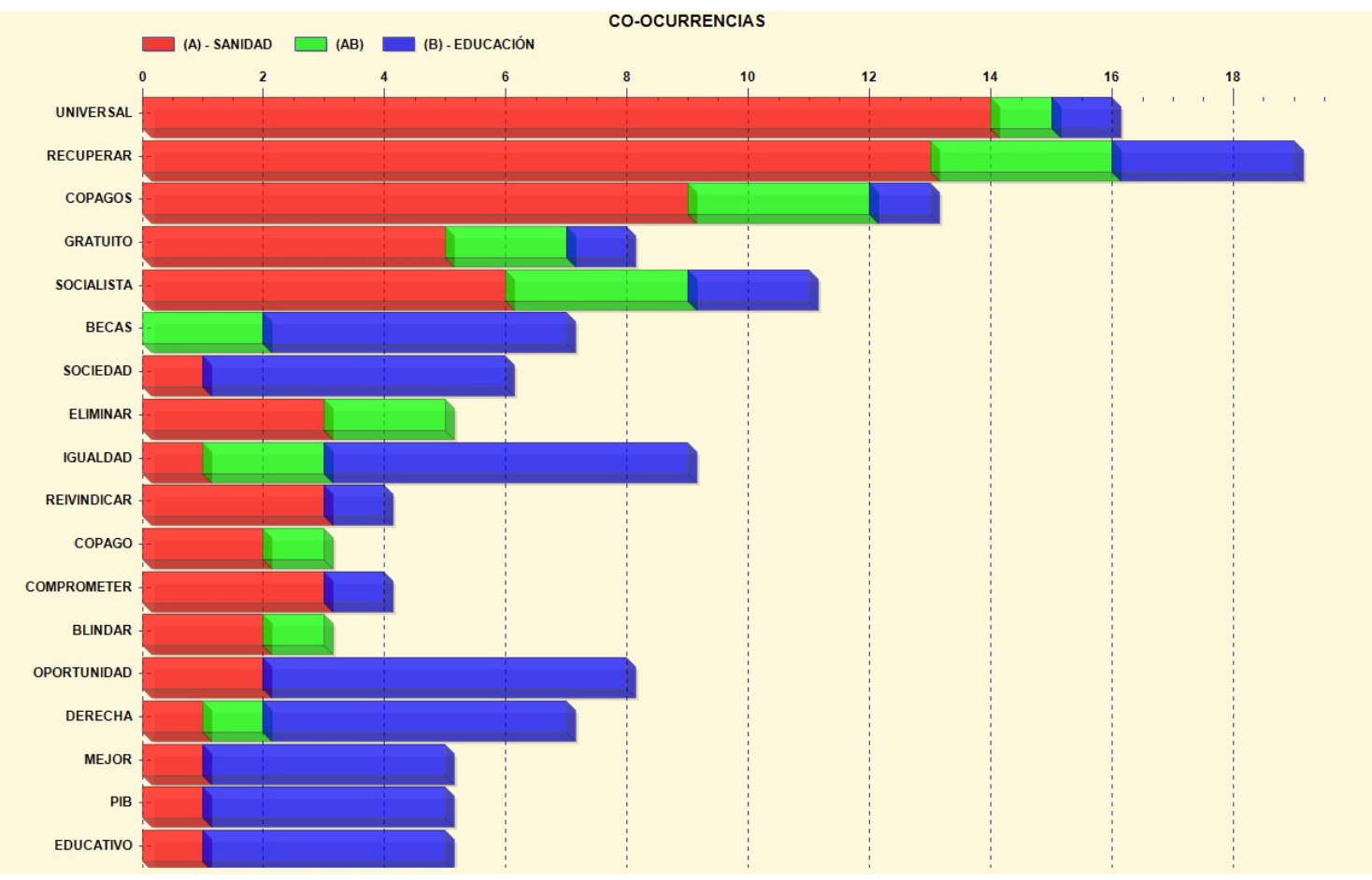

Fuente: Elaboración propia

En el caso de Albert Rivera, candidato del partido de Ciudadanos, aparecen un total de 13 lemas co-ocurrentes (gráfico 5). De estos, en tan solo 5 se ha encontrado una co-ocurrencia con la palabra "sanidad", mientras que 12 son las co-ocurrentes con ambas palabras clave y 9 relacionadas únicamente con "educación". Aun así, cabe mencionar que el máximo de co-ocurrencias ha sido de 5 , que sucede en tres ocasiones, con "gratuito", "ciudadano" y "vía". Por otra parte, es en "igualdad" y "vía" donde más co-ocurrencias existen entre ambas palabras clave (3 veces cada una). Es significativo también que la palabra que más veces co-ocurre con "sanidad" sea "entrevista" (3 veces), seguida de "paro" (2 veces). 
Gráfico 5: Co-ocurrencia entre "Sanidad" y "Educación" en el discurso de Albert Rivera

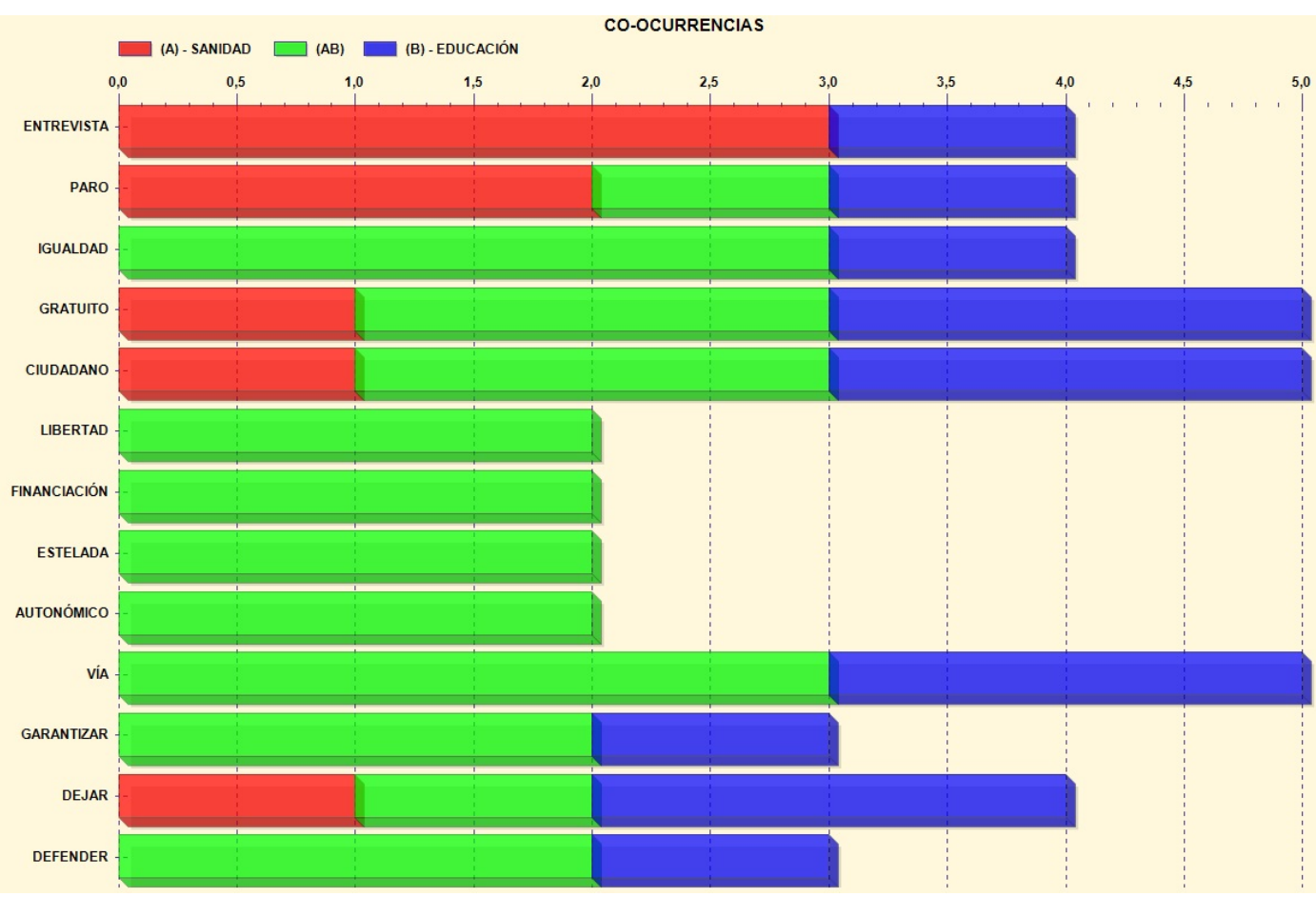

Fuente: Elaboración propia

Si nos fijamos en el discurso de Pablo Iglesias (gráfico 6), candidato de Podemos, encontramos un total de 13 palabras co-ocurrentes con el binomio analizado. De ellas, 11 están vinculadas con "sanidad", siendo la más utilizada de todas las palabras "público" (7 veces), seguida de "servicio" y "entrevista" (3 veces cada una). El margen de palabras que co-ocurren con ambas palabras clave es inferior, siendo de 1 vez en casi todas las ocasiones.

Gráfico 6: Co-ocurrencia entre "Sanidad" y "Educación" en el discurso Pablo Iglesias

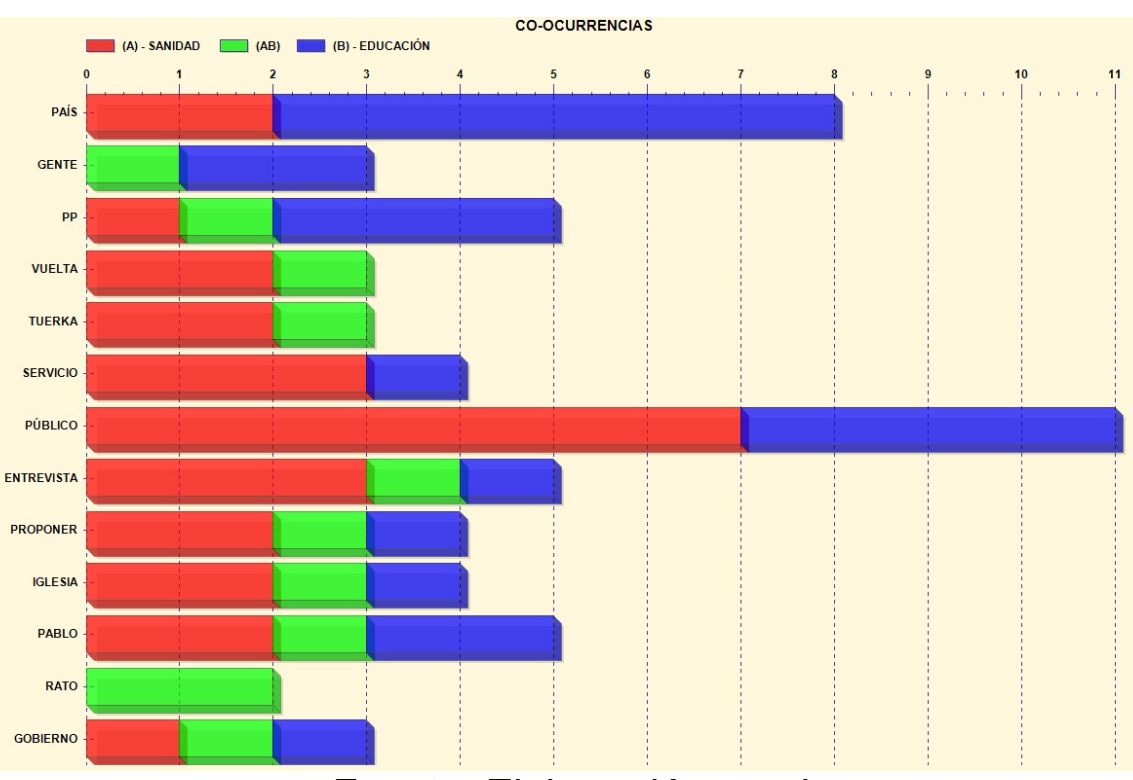

Fuente: Elaboración propia 
Finalmente, los resultados del análisis del contenido de los tuits de Alberto Garzón, candidato de Unidad Popular, muestran 7 palabras resultantes de la correlación, de las cuales 5 están co-ocurriendo con "sanidad". No obstante, cabe mencionar que el máximo de repeticiones que encontramos en sanidad es de 2 veces, como se puede ver en los casos de "público", "PP" y "derecho". Mientras que en el caso de la co-ocurrencia con "educación", el número máximo se eleva ligeramente siendo "público" la palabra con más frecuencia (5 veces). Respecto a los términos resultantes de la co-ocurrencia de ambas palabras clave, la frecuencia máxima de repetición es de dos veces para los términos "pensión" y "decidir").

Gráfico 7: Co-ocurrencia entre "Sanidad" y "Educación" en el discurso de Alberto Garzón

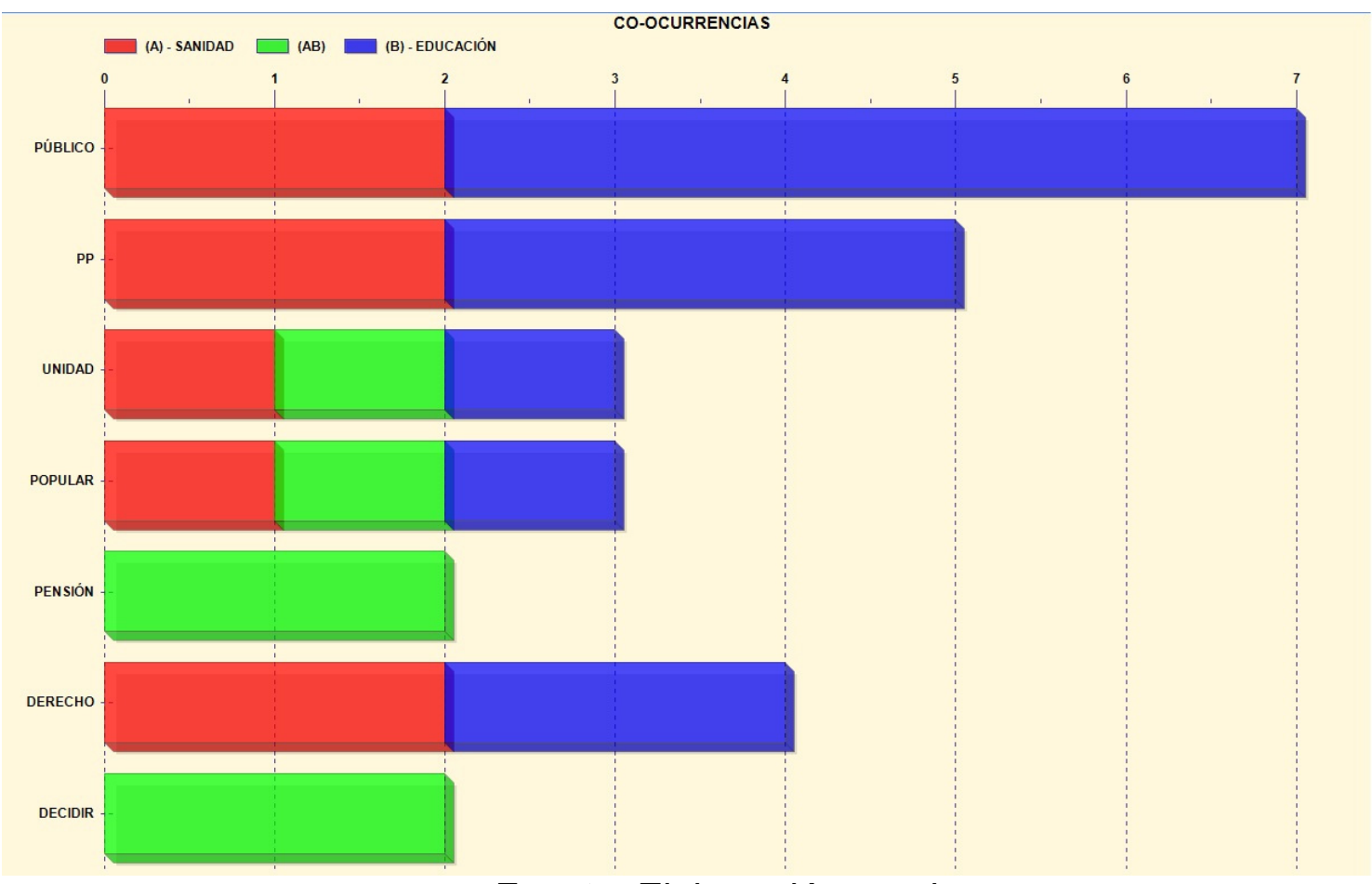

Fuente: Elaboración propia.

\subsection{Análisis de contenido de los programas electorales de los cinco partidos más votados}

El estudio de estos documentos ha sido a través de la versión completa en PDF que todos los partidos han subido a su página web oficial. No obstante, también es de destacar las dificultades que presentan algunos programas al estar maquetados de forma que no se puede "copiar" y "pegar" o "subrayar" el texto, característica que también afecta a la búsqueda por palabras en el documento, como ha sido el caso de Ciudadanos y Unidad Popular.

Asimismo, es necesario destacar la no uniformidad que presentan los programas electorales de los partidos estudiados. Las secciones no son fijas y la pormenorización con la que se desarrollan los programas tampoco. Los siguientes apartados muestran los diferentes modelos de exposición de la información en los programas electorales de las formaciones políticas seleccionadas para el presente estudio. 


\subsubsection{Partido Popular}

El programa electoral presentado por el Partido Popular para las elecciones Generales de 2015 se titula "Seguir Avanzando". Contiene un total de 226 páginas, de las cuales 13 páginas son las que desarrollan la parte específica del programa electoral en materia de sanidad (un 5'7\% del programa). Esta sección está indexada dentro del tercer bloque (de seis), titulado "Primero las personas", en el segundo subapartado (de seis): "Mejorando nuestro sistema sanitario".

La parte que desarrolla la sanidad en el programa está compuesta por dos grandes bloques:

1) "Nuestro compromiso", en el que exponen sus convicciones sobre lo que es y esperan que sea el sistema sanitario español.

2) "Nuestro proyecto", compuesto por 47 propuestas divididas en ocho apartados.

Asimismo, también incluye medidas relativas a la sanidad en dos apartados independientes del específico. En concreto, en "La familia, el centro de nuestra sociedad", en el que se desarrolla un plan sobre estrategias de atención al Parto Normal y de Salud Reproductiva. Asimismo, en el apartado "Nuestros mayores: el valor de la experiencia con pensiones garantizadas" se desarrolla el programa de la Dependencia, el plan de prevención de caídas y la incorporación de los geriatras en los centros de salud.

De los campos de análisis que buscábamos para esta investigación (Privatización, Servicio Público, Cuidados Paliativos, Regulación, Financiación, Medicina Preventiva, Profesionales sanitarios, Investigación y Terapias no convencionales), se han quedado sin recoger Privatización, Cuidados Paliativos y Terapias no convencionales, y de los que han sido tratados, el desarrollo ha sido bastante superficial.

Respecto a las terapias no convencionales, a pesar de su ausencia de protagonismo en el programa electoral, podríamos relacionar las siguientes afirmaciones con estas terapias, aunque no está expuesto de ninguna forma evidente.

\subsubsection{Partido Socialista Obrero Español}

El programa electoral presentado por el Partido Socialista Obrero Español (PSOE) para las elecciones Generales de 2015 contiene un total de 274 páginas, de las cuales 13 están dedicadas a la sanidad ( $47 \%$ del programa). La sanidad está integrada en el bloque denominado "El Estado Social", esto es debido a que, según el PSOE (2015) la Sanidad es una de las necesidades básicas para garantizar un sistema de protección social. Es el sexto apartado, de doce que componen este bloque y se desarrolla en los tres puntos, de los cuales es el tercero el que contiene las propuestas del partido para las elecciones. Entre ellas destaca incorporar medidas preventivas, realizar una política de salud transversal, entender la sanidad como inversión, reforzar las estrategias en cáncer y cuidados paliativos, mejorar la inversión para la formación del personal profesional sanitario y para la investigación, fomentar una cultura de la salud, dar acceso a los ciudadanos a su historial electrónico y regular la muerte digna.

Los campos que buscábamos en esta investigación han sido tratados todos por el PSOE en su programa, con mayor o menor desarrollo. Respecto a las terapias no 
convencionales, literalmente, solo están presentes en un párrafo, dentro de las propuestas que responden al objetivo "La Salud en todas las políticas", en el que proponen:

"Regular, desde el consenso, el sector de las terapias naturales, que permita al ciudadano acudir a estos técnicos con las debidas garantías" (Partido Socialista Obrero Español, 2015, p. 204)

En esta última cita podemos ver la nomenclatura que el PSOE utiliza para referirse a las terapias no convencionales: "Terapias naturales"

\subsubsection{Ciudadanos}

El programa electoral presentado por Ciudadanos para las elecciones Generales de 2015 se titula "El nuevo proyecto común para España". Contiene un total de 338 páginas, de las cuales 15 páginas son las que desarrollan su programa relativo a la sanidad (4'4\% del programa). La sanidad está indexada en el quinto punto (de diecisiete) del programa, bajo el título "Nuestro sistema sanitario". Esta parte está compuesta por 16 subapartados.

Los campos que buscábamos en esta investigación han sido tratados todos en su programa, con mayor o menor desarrollo. A pesar de que sería perfectamente esperable buscar el posicionamiento del partido y sus intenciones respecto a las terapias no convencionales en el sexto apartado (Políticas de Salud), en el décimo (Investigación y evaluación de tecnologías y medicamentos, e incorporación a la cartera de servicios del Sistema Nacional de Salud), en el decimoquinto apartado (Un modelo de farmacia comunitaria asistencial) o en el decimosexto (Medidas conjuntas a nivel de Farmacia), estas no son abordadas en profundidad en ninguno de ellos, tan sólo se hace referencia a este tipo de terapias o medicamentos no convencionales en dos ocasiones puntuales, en la introducción del apartado sanitario con la siguiente afirmación:

"El Sistema Nacional de Salud debe incorporar en su cartera los servicios que hayan demostrado su seguridad y eficacia, después de una evaluación científica y económica, que ponga a disposición de los ciudadanos los medios diagnósticos y los tratamientos realmente resolutivos y eficientes." (Ciudadanos, 2015, p. 161)

Y en el apartado "Investigación y evaluación de tecnologías y medicamentos, e incorporación a la cartera de servicios del Sistema Nacional de Salud", concretamente con la afirmación:

"Implementaremos una estrategia a largo plazo de reinversión que contemple no solo la incorporación de nuevas tecnologías sanitarias, sino también la desinversión en aquellas que no sean coste-efectivas. Así como regularemos la eliminación de aquellas estrategias o practicas clínicas desaconsejadas por la evidencia científica." (Ciudadanos, 2015, p. 170)

En esta última cita podemos ver la nomenclatura que Ciudadanos utiliza para referirse a las terapias no convencionales: "Estrategias o prácticas clínicas desaconsejadas por la evidencia científica".

Sorprendentemente, Ciudadanos explica que eliminará este tipo de terapias por no existir investigaciones que demuestren una evidencia científica de la efectividad de este tipo de técnicas, pero sí que regula el cannabis para asegurarse que las personas que necesiten consumir este tipo de sustancia tengan aseguradas un control de calidad. 


\subsubsection{Podemos}

El programa electoral presentado por Podemos para las elecciones Generales de 2015 se titula "Queremos, Sabemos, Podemos. Un programa para cambiar nuestro país". Contiene un total de 332 páginas, de las cuales en 15 de ellas aborda medidas relacionadas con el sector sanitario (4'5\% del programa electoral). El apartado específico sobre Sanidad está indexado en el tercer bloque (de los nueve que lo componen), denominado "Democracia social" y el apartado específico "Sanidad". Está compuesto por 15 medidas (de la propuesta 114 a la propuesta 128 del programa, ambas incluidas), sin ningún tipo de categorización interna, siguiendo el estilo de todo el programa.

No obstante, independientemente de este apartado específico, debido a la visión concreta de Podemos de la sanidad, que entiende que se debe "incorporar la salud como cuestión transversal a todas las políticas públicas" (Podemos, 2015, p. 78), también se abordan aspectos sanitarios en los siguientes apartados: "Dependencia y Diversidad funcional" (Ley de la dependencia, rehabilitación y fisioterapia gratuitas para las personas en situación de dependencia o de diversidad funcional; aumento y agilización de las prestaciones por material ortoprotésico), "Igualdad" (Derecho a una maternidad libre y decidida, acceso a la reproducción asistida, lucha contra el VIH), "Justicia y Derechos Humanos" (Despenalización del cannabis) y "Migraciones y asilo" (Cobertura sanitaria universal).

De los campos que buscábamos en esta investigación, se han mencionado Servicio Público, Regulación, Financiación e Investigación, mientras que Privatización, Cuidados Paliativos, Medicina preventiva y Terapias no convencionales no han sido temáticas consideradas en el programa electoral del partido.

A pesar de que entre las 15 medidas que promulgan se encuentran cuestiones tan específicas como la prohibición del uso de bisfenol A (Podemos, 2015: 76), un producto contaminante en la alimentación, y tan generales como la ampliación de la cartera de medicamentos financiados (Podemos, 2015, p. 75). Sobre las terapias no convencionales y los medicamentos y utensilios necesarios para ello no se encuentra ninguna medida relacionada, ni que lo aborde.

\subsubsection{Unidad Popular}

El programa electoral presentado por Unidad Popular para las elecciones Generales de 2015 contiene un total de 103 páginas, de las cuales 13 están dedicadas a la sanidad (12'6\% del programa). El apartado específico de sanidad está integrado en el punto 4 "Derechos sociales", en el subapartado segundo. Está compuesto por ocho apartados. Asimismo, encontramos más propuestas relacionadas en otras secciones: en el punto 3 "Medio Ambiente y Modelo sostenible", dentro del apartado "Regulación del consumo", se propone el acceso terapéutico al cannabis. $Y$ en el punto 5 "Derechos Humanos", en el apartado "Derechos sexuales y reproductivos", se abordan las propuestas propias de la salud sexual.

Los campos que buscábamos para abordar esta investigación han sido tratados todos por Unidad Popular en su programa, con mayor o menor desarrollo, a excepción del tema relativo a los Cuidados Paliativos, del que no se menciona ninguna medida. Respecto a las terapias no convencionales, proponen una política farmacéutica integral que atienda las necesidades reales de la población "dejando 
de financiar y/o prohibiendo la inclusión en la categoría de fármacos productos de nula utilidad o que solo demuestran servir para crear falsas necesidades y expectativas en la población con un fin lucrativo" (Unidad Popular, 2015, p. 55). Es por ello que afirman que "solamente se financiarán tratamientos basados en la evidencia y la promoción del bienestar físico, mental y social de las personas, rechazando aquellas propuestas terapéuticas que no han demostrado ser eficaces para las diferentes enfermedades o trastornos hasta que existan pruebas sólidas que demuestren esta eficacia." (Unidad Popular, 2015, p. 56). De hecho, también proponen regular la publicidad de medicamentos para evitar estrategias de comunicación basadas en beneficios que no se ajustan a la evidencia científica.

Tabla 1

Tratamiento de las terapias no convencionales por los partidos

\begin{tabular}{|c|c|c|c|}
\hline Partido & $\begin{array}{l}\text { Terapias No } \\
\text { Convencionales }\end{array}$ & Nombre & Observaciones \\
\hline PP & 凶 & - & - \\
\hline PSOE & $\nabla$ & "Terapias naturales" & $\begin{array}{l}\text { Quiere regularlas } \\
\text { para poder } \\
\text { garantizar la } \\
\text { calidad }\end{array}$ \\
\hline Ciudadanos & $\nabla$ & $\begin{array}{l}\text { "Estrategias o } \\
\text { prácticas clínicas } \\
\text { desaconsejadas por } \\
\text { la evidencia científica" }\end{array}$ & $\begin{array}{l}\text { Quiere eliminarlas } \\
\text { de la cartera de } \\
\text { servicios del SNS }\end{array}$ \\
\hline Podemos & 冈 & - & - \\
\hline $\begin{array}{l}\text { Unidad } \\
\text { Popular }\end{array}$ & $\nabla$ & $\begin{array}{l}\text { "Procedimientos } \\
\text { terapéuticos } \\
\text { pseudocientíficos" }\end{array}$ & $\begin{array}{l}\text { Quiere eliminarlas } \\
\text { de la cartera de } \\
\text { servicios del SNS }\end{array}$ \\
\hline
\end{tabular}

Fuente: Elaboración propia

\section{DISCUSIÓN Y CONCLUSIONES}

Los resultados del análisis de los mensajes emitidos por los candidatos en Twitter durante la campaña electoral, nos muestra que la sanidad no ha sido uno de los temas prioritarios en campaña. Como podemos ver en el gráfico 8, es Pedro Sánchez el candidato que más veces ha mencionado en su discurso la palabra sanidad, mientras que Mariano Rajoy es el que menos ocasiones lo ha hecho. Albert Rivera es quien destaca en la asociación de ideas sobre la sanidad y la educación; y Alberto Garzón es el candidato que menos asociaciones de interdependencias discursivas ha realizado entre la sanidad y la educación. 
Gráfico 8: Recuento de co-ocurrencias de palabras

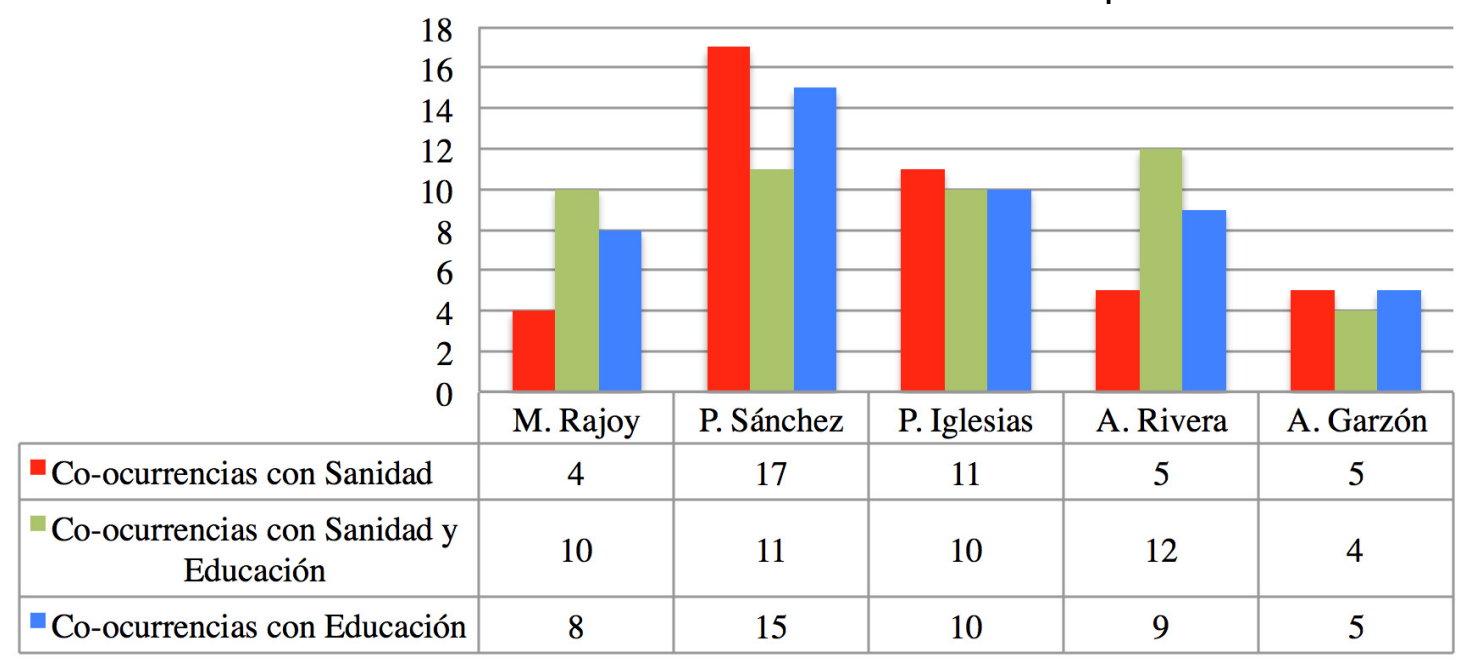

Fuente: Elaboración propia.

Si nos centramos en las palabras que surgen como co-ocurrencia con el término "sanidad", encontramos que el discurso de Rajoy co-ocurre con "gobierno", "mejor" y "público", lo que podemos interpretar como un discurso sanitario apoyado en una mejora del servicio público. Por otra parte, Sánchez habla de sanidad universal, de eliminar copagos y recuperar el sistema anterior. Mientras que Iglesias también habla de servicio público. Por su parte, Rivera relaciona la sanidad y el paro, y ensalza el valor de lo gratuito. Finalmente, Garzón habla de la sanidad como derecho, como servicio público y menciona en su discurso al partido que está en el gobierno.

En lo que respecta a los programas electorales, como se puede ver en la gráfica 10 , el partido que más espacio le ha dedicado a la sanidad ha sido Unidad Popular (con un $12{ }^{\prime} 6 \%$ del programa), seguido por el Partido Popular (con un $5^{\prime} 7 \%$ ), el PSOE (con un $4{ }^{\prime} 7 \%$ ), Podemos (con un 4'5\%) y Ciudadanos (con un 4'4\%). El resultado tan positivamente desmarcado de Unidad Popular frente al resto de partidos destaca notablemente no sólo por el espacio dedicado, sino por el contraste con el discurso mantenido en Twitter por Alberto Garzón, cuya cuota dedicada a la sanidad es de las más inferiores.

Gráfico 9: Porcentaje del programa electoral dedicado a la sanidad

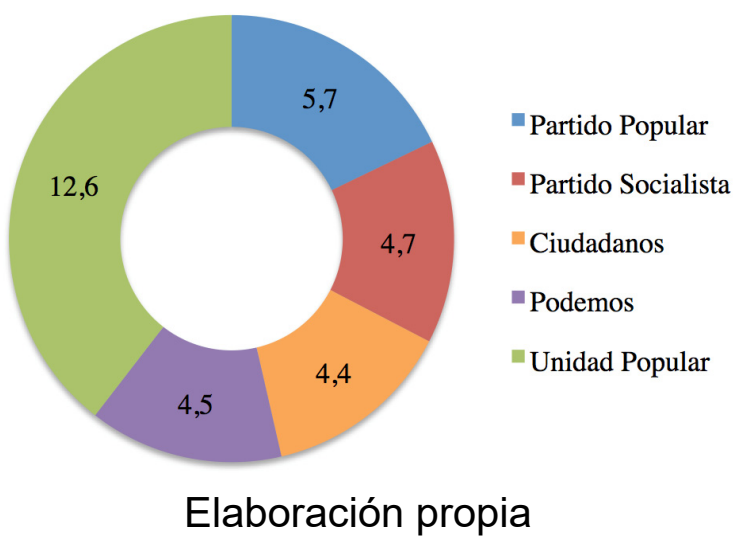

En cuanto a la validación de hipótesis, los resultados refutan la primera. Se ha podido comprobar cómo la repercusión de la sanidad en el discurso de los candidatos en Twitter no ha alcanzado protagonismo suficiente como para ser un 
punto clave en su agenda temática. La segunda hipótesis queda validad por completo, debido a que el PSOE, partido escorado a la izquierda, representa la formación política que más cuota tiene respecto al tema sanitario; y el PP, escorado a la derecha, es el que menos mensajes ha emitido sobre sanidad.

No obstante, la tercera hipótesis queda corroborada en parte, puesto que, efectivamente, la representación de las terapias no convencionales en el discurso de los candidatos en Twitter es nula, pero en contraposición a lo que se esperaba, su desarrollo en el programa electoral no ha sido precisamente extenso, puesto que a lo máximo que han llegado los partidos ha sido a mencionarlas.

A modo de conclusión resumiremos que, en general, la sanidad no representa una prioridad ni en discurso digital de los candidatos, ni en el programa electoral. Por las publicaciones en Twitter es Pedro Sánchez, candidato del PSOE, quien más espacio le ha dedicado a este tema. Mientras que quien ha dedicado más porcentaje de su programa electoral al sistema sanitario ha sido Unidad Popular.

Respecto a las terapias no convencionales, no sólo no han aparecido en el flujo de discursos en Twitter sino que su presencia es muy escasa en los programas electorales. Tan solo hacen referencia a estas Unidad Popular, refiriéndose a ellas como "Procedimientos terapéuticos pseudocientíficos" (2015, p. 56); el PSOE, denominándolas "Terapias naturales" (2015, p. 204) y Ciudadanos, que las define como "Estrategias o prácticas clínicas desaconsejadas por la evidencia científica" (2015, p. 170). Es decir, de estos tres partidos que sí que se posicionan en su programa electoral, Unidad Popular y Ciudadanos sí que quieren eliminarlos de la cartera de servicios del sistema nacional de salud, mientras que el PSOE propone regularlo para poder garantizar la calidad de estas terapias para las personas que quieran utilizarla.

\section{NOTAS}

${ }^{1} 10.000$ millones menos para sanidad http://politica.elpais.com/politica/2015/03/14/actualidad/1426369300 405355.html ${ }^{2}$ La OMS, a causa de la multiplicación de las reacciones adversas derivadas de tratamientos no convencionales, señala la escasa regulación a nivel mundial y la necesidad de informar a los ciudadanos http://www.who.int/mediacentre/news/releases/2004/pr44/es/

${ }^{3}$ REAL DECRETO 2208/1994, de 16 de noviembre, por el que se regula los medicamentos homeopáticos de uso humano de fabricación industrial (https://www.boe.es/boe/dias/1994/11/28/pdfs/A36299-36301.pdf)

${ }^{4}$ REAL DECRETO 1277/2003, de 10 de octubre, por el que se establecen las bases generales sobre autorización de centros, servicios y establecimientos sanitarios (https://www.boe.es/boe/dias/2003/10/23/pdfs/A37893-37902.pdf)

${ }^{5} \mathrm{El}$ informe fue publicado en diciembre de 2011. Se puede consultar aquí: http://www.msssi.gob.es/novedades/docs/analisisSituacionTNatu.pdf

${ }^{6}$ http://www.msssi.gob.es/normativa/docs/Ordenhomeopaticos.pdf

7 Herramienta de análisis de Twitter (Borra \& Rieder, 2014) que permite descargar los mensajes emitidos por aquellas cuentas que tienen configurado el perfil público en la red social.

8 Resultados de las Elecciones Generales de 2015. Fuente: El País http://resultados.elpais.com/elecciones/2015/generales/congreso/

${ }^{9}$ El análisis de las publicaciones de los cabezas de lista supondría un estudio parcial en cuanto a los actores participantes en la comunicación política, aunque tanto 
medios como ciudadanos tienen en parte protagonismo "en relación con la actividad desarrollada por los candidatos" (López García et al., 2015:220). Siendo conscientes de este sesgo, se escoge el discurso en Twitter de estos actores para poder medir así en este sector concreto la repercusión de la sanidad.

${ }^{10}$ La página web oficial donde es explicada de forma pormenorizada este análisis es la siguiente: https://tlab.it/es/allegati/help es online/mcoppie.htm

${ }^{11}$ La separación del texto en fragmentos la realiza de forma automática el software, para ello tiene como referencia el punto y a parte para dividir el texto. Previamente al procesamiento del texto por el software, se ha tenido que preparar el corpus para obtener una interpretación correcta por el programa informático. Para ello ha sido necesario agrupar todos los tuits en un archivo .txt, separados por el signo que corresponde.

\section{REFERENCIAS BIBLIOGRÁFICAS}

Ballvé Moreno, J. L. (2003). “¿Quién utiliza las medicinas no convencionales y por qué?" Humanitas, Humanidades Médicas, 1, 31-40.

Borra, E., \& Rieder, B. (2014). "Programmed method: developing a toolset for capturing and analyzing tweets", Aslib Journal of Information Management, 66(3), 262-278.

Cairo, A. (2013). The functional art. An introduction to information graphics and visualization. Berkeley: New Riders.

Ciudadanos (2015). El nuevo proyecto común para España. Programa electoral elecciones generales 2015. Recuperado de https://www.ciudadanoscs.org/var/public/sections/page-programa-electoral-20d/programa-electoral.pdf

Cuesta, U., y Gaspar, S. (2013). Aspectos psicosociales, éticos y normativos de la reputación on line. Nueva Época, 14, 58-68.

Cuesta, U., Ugarte, A., Gaspar, S., y Menéndez, T. M. (2011). Comunicación social institucional y salud: El caso de Madrid Salud del Ayuntamiento de Madrid. En II Congreso Internacional Ciudades Creativas.

Idoyaga Molina A. (2000). La calidad de prestaciones de salud desde el punto de vista del usuario. Scripta Ethnologica, 22, 21-85.

Ministerio de Sanidad, Política Social e Igualdad (2011). Terapias naturales. Recuperado

de http://www.msssi.gob.es/novedades/docs/analisisSituacionTNatu.pdf

Palomo Torres, M. B. (2014). La (r)evolución social del periodista. La credibilidad como desafío vital. Telos: Cuadernos de comunicación e innovación, 98, 104114.

Podemos (2015). Queremos, sabemos, podemos. Un programa para cambiar nuestro país. Elecciones generales 20 de diciembre de 2015. Recuperado de http://unpaiscontigo.es/wp-content/plugins/programa/data/programa-es.pdf

PP (2015). Seguir avanzando. 2016-2020. Programa electoral para las elecciones generales de 2015.2 Recuperado de http://www.pp.es/sites/default/files/documentos/programa2015.pdf

PSOE (2015). El cambio que une. Programa electoral elecciones generales 2015. Recuperado content/2015/11/PSOE Programa Electoral 2015.pdf

http://www.psoe.es/media- 
Unidad Popular (2015). Programa participativo generales 2015. Recuperado de http://unidadpopular.es/wpcontent/uploads/2015/12/Programa participativo UP 2015.12.06.pdf

Valera Ordaz, L. (2014). El debate público en la blogosfera política española durante la campaña electoral de 2011: ¿Hacia un espacio público enriquecido o fragmentado?. Trípodos, 1(34), 153-170.

Yau, N. (2011). Visualize This: The FlowingData Guide to Design, Visualization, and Statistics. Hoboken, NJ: John Wiley \& Sons. 